\title{
Original Research \\ Endoplasmic reticulum stress as a novel target to inhibit transdifferentiation of human retinal pigment epithelial cells
}

\author{
Sha Ouyang ${ }^{1,2,3,4}$, Dan $\mathrm{Ji}^{2,3}$, Shikun $\mathrm{He}^{4, *}$, Xiaobo Xia ${ }^{2,3, *}$ \\ ${ }^{1}$ Department of Ophthalmology, The Third Xiangya Hospital, Central South University, 410000 Changsha, Hunan, China \\ ${ }^{2}$ Eye Center of Xiangya Hospital, Central South University, 410008 Changsha, Hunan, China \\ ${ }^{3}$ Hunan Key Laboratory of Ophthalmology, 410008 Changsha, Hunan, China \\ ${ }^{4}$ Department of Pathology, USC Roski Eye Institute, Keck School of Medicine of the University of Southern California, Los Angeles, CA 90033, USA \\ *Correspondence: xbxia21@csu.edu.cn (Xiaobo Xia); shikunhe@usc.edu (Shikun He) \\ Academic Editors: Graham Pawelec \\ Submitted: 31 August 2021 Revised: 7 December 2021 Accepted: 12 December 2021 Published: 24 January 2022
}

\begin{abstract}
Background: The epithelial to mesenchymal transition (EMT) of retinal pigment epithelial (RPE) cells is a critical event in the pathogenesis of proliferative vitreoretinopathy and neovascular age-related macular degeneration, which are the leading causes of severe vision loss. Endoplasmic reticulum (ER) stress has been implicated in the EMT of many cell types and various ocular diseases. However, the relationship between ER stress and EMT in RPE cells remains unknown. Therefore, in the study, we explored the impact of ER stress on EMT in RPE cells. Methods: Different concentrations of tunicamycin (TM) and thapsigargin (TG) were used to induce ER stress in human RPE cells. The expression of epithelial marker, mesenchymal markers and some of genes/proteins involved in TGF- $\beta /$ Smad signaling were analized by qPCR, western blot or immunostaining at the condition with or without stimulation of TGF- $\beta 2$ (10 ng/mL). Boyden chamber and scratch assay were used to evaluate the migration of RPE cells, while cell viability and apoptosis of RPE cells were measured by MTT and TUNEL assay, respectively. Results: Treatment of RPE cells with TM and TG ( $24 \mathrm{~h})$ reduced the expression of $\alpha$-SMA and FN, and increased the expression of Occludin in a dose dependent manner at protein level, which was highly associated with the expression of GRP78. Treatment with TGF- $\beta 2$ significantly increased the expression of $\alpha$-SMA and FN, and decreased the expression of Occludin both in protein and mRNA levels, which was significantly inhibited by a $4 \mathrm{~h}$ pre-treatment with TM. In addition, the expression of TGF- $\beta$ RII and Smad2/3, and mRNAs of TGF- $\beta$ RII and Smad3 were also decreased by the TM treatment. TM-induced ER stress inhibited RPE cell migration, and high concentrations of TM and TG reduced cell viability and induced apoptosis of RPE cells. Conclusions: Chemical induction of ER stress inhibited EMT and migration in RPE cells, possibly by inactivation of TGF- $\beta$ signaling, suggesting that regulation of ER stress in RPE cells may be a new approach to prevent the development of intraocular fibrosis.
\end{abstract}

Keywords: Endoplasmic reticulum stress; Epithelial-mesenchymal transition; Cell movements; TGF- $\beta$ signaling; Retinal pigment epithelial cells

\section{Introduction}

Intraocular fibrotic diseases, such as proliferative vitreoretinopathy (PVR), late-stage neovascular age-related macular degeneration (nAMD), and proliferative diabetic retinopathy (PDR), can occur in response to ocular trauma, ischemia, inflammation, and degenerative disease. These diseases usually lead to severe visual impairment or irreversible vision loss in the majority of patients. The formation of subretinal, epiretinal, or intravitreal fibrotic membranes due to extensive wound healing responses has been identified as an important step in the pathogenesis of these fibrotic diseases. This process orchestrates the interaction of several cellular components, including RPE cells, glial cells, fibroblasts, and inflammatory cells [1], with many inflammatory cytokines and growth factors, resulting in overproduction or remodeling of the extracellular matrix (ECM). Among all these involved cell types, RPE cells have been recognized to play a critical role in the development of retinal fibrosis $[2,3]$.
Epithelial to mesenchymal transition (EMT) is a biological process that refers to the phenomenon in which epithelial cells are converted into mesenchymal cells under specific physiological and pathological conditions. In this process, epithelial cells lose their cell-cell junctions and apical-basal polarity, reorganize their actin cytoskeleton, and gain cell motility and invasion properties. This is characterized by the downregulation of epithelial molecular markers, such as occludin, zonula occludens-1 (ZO-1), and E-cadherin, and upregulation of mesenchymal molecular markers, such as $\alpha$-SMA and fibronectin (FN). Accumulating evidence has revealed the pivotal role of EMT in wound healing, tissue reconstruction, and fibrosis, including the formation of fibrotic lesions on the retina $[4,5]$. In the development of PVR and at the end-stage of nAMD, with the stimulation of various growth factors and cytokines, the RPE cells, which are a monolayer of highly polarized hexagonal cells under physiological conditions, undergo EMT. This enables them to lose their epithelial characteristics, transdifferentiate into fibroblast-like cells, and migrate 
to the subretinal space, resulting in the formation of a fibrotic membrane after rhegmatogenous retinal detachment in PVR or choroidal neovascularization in AMD [6,7].

ER stress has long been studied for its role in neurodegenerative diseases and diabetes, including AMD and PDR. During the past 10 years, accumulating evidence has indicated the potential role of ER stress in a variety of fibrotic diseases, including fibrosis of the liver, heart, kidney, and lung $[8,9]$. The ER is a multifunctional cellular organelle that is essential for maintaining protein homeostasis. Accumulation of misfolded/unfolded proteins in the ER lumen leads to activation of the unfolded protein response (UPR), a process known as ER stress, with the release of a critical ER stress regulator, glucose-regulated protein 78/binding immunoglobulin protein (GRP78/BiP). Recently, several studies reported the association between ER stress and EMT in regulating fibrosis development in various cell types, such as pulmonary epithelial cells, peritoneal mesothelial cells, and human lens epithelial cells [10-12]. However, the effects of ER stress on EMT in RPE cells have not been studied.

In this study, we conducted a series of experiments to investigate the impact of ER stress on the process of EMT in the RPE cell.

\section{Materials and methods}

\subsection{Retinal pigment epithelial cell culture}

Human RPE cells were isolated from postmortem human fetal eyes (gestational age, 16-18 weeks) obtained from Advanced Bioscience Resources Inc. (Alameda, CA, USA) and cultured as previously described [13]. Briefly, cells were cultured in DMEM containing $2 \mathrm{mM}$ Lglutamine, $100 \mathrm{U} / \mathrm{mL}$ penicillin, $100 \mu \mathrm{g} / \mathrm{mL}$ streptomycin, and $10 \%$ heat-inactivated fetal bovine serum (FBS). Cultured human RPE cells were used from passage 2 to 4 . Cells were confirmed to be RPE by their typical morphology, their immunoreactivity for cytokeratin (>95\%), and the lack of immunoreactivity for macrophage or endothelial cell markers.

\subsection{Western blotting}

RPE cells were lysed in lysis buffer (RIPA buffer, Thermo Fisher, USA), and the extracted cell lysates were separated using Tris-HCl/Tris-Glycine gels (Ready Gel; Bio-Rad, Hercules, CA, USA). The proteins were then transferred to polyvinylidene fluoride microporous membranes (Millipore, Billerica, MA, USA). The blots were blocked with $5 \%$ milk for $1 \mathrm{~h}$ at room temperature and incubated with the corresponding primary antibodies (Supplementary Table 1) overnight at $4{ }^{\circ} \mathrm{C}$. Blots were washed and incubated with horseradish peroxidaselabeled secondary antibodies (Vector Laboratories, Inc. Burlingame, CA, USA) for $1 \mathrm{~h}$ at room temperature. After addition of chemiluminescent detection solution (ECL Western Blotting Substrate; Thermo Fisher, Waltham, MA,
USA), images were developed using an enhanced chemiluminescence detection system (ChemiDoc ${ }^{\mathrm{TM}} \mathrm{XRS}+$ System, Bio-Rad). Glyceraldehyde-3-phosphate dehydrogenase (GAPDH) served as the protein-loading control. Densitometry was performed based on three independent experiments using Image J software (Image J 1.50i bundled with 64-bit java 1.8.0) (http://imagej.nih.gov.libproxy1.usc.edu/ ij/; National Institutes of Health, Bethesda, MD, USA).

\subsection{RNA isolation and real-time RT-qPCR}

Total RNA was isolated using a RNeasy mini kit (QIAGEN, Valencia, CA, USA), and cDNA was synthesized from total RNA using the ImProm-IITM Reverse Transcription System (Promega, Madison, WI, USA). Real-time PCR was performed in duplicate using a commercial SYBR Green master mix (Roche, Basel, Switzerland) with a LightCycler 480 (Roche) according to the manufacturer's protocols. The cycling conditions for the RT-qPCR were as follows: pre-incubation at $95{ }^{\circ} \mathrm{C}$ for $5 \mathrm{~min}$, followed by 45 cycles of denaturing at $95{ }^{\circ} \mathrm{C}$ for $10 \mathrm{~s}$, annealing at $55{ }^{\circ} \mathrm{C}$ for $10 \mathrm{~s}$, and extension at $72{ }^{\circ} \mathrm{C}$ for $10 \mathrm{~s}$. The primer sequences are shown in Supplementary Table 2. Relative changes in mRNA expression were determined by calculating the $\Delta \Delta \mathrm{CT}$ (cycle threshold) values. mRNA levels were normalized relative to GAPDH mRNA and reported as fold-change over controls.

\subsection{Immunofluorescence staining and immunohistochemistry}

RPE cells were cultured in 4-chambered culture slides (Falcon, Thermo Fisher). The cells were rinsed in phosphate-buffered saline (PBS) for $3 \mathrm{~min}$, fixed with $4 \%$ paraformaldehyde for $10 \mathrm{~min}$, and permeabilized with $0.1 \%$ Tween 20 (Bio-Rad) in PBS. After 30-min blocking with $5 \%$ normal goat serum, the cells were incubated with the primary antibodies (Supplementary Table 1) overnight at $4{ }^{\circ} \mathrm{C}$ and then incubated with the corresponding secondary antibodies for $30 \mathrm{~min}$ at room temperature. All antibodies were diluted in PBS. The slides were mounted with mounting medium containing DAPI (Vector Laboratories) and examined with a KEYENCE fluorescence microscope (BZX700, San Diego, CA, USA).

\subsection{MTT assay}

Human RPE cells were cultured in DMEM containing $10 \%$ FBS in a 96-well plate and then treated with different concentrations of TM (10-200 ng/mL) for $24 \mathrm{~h}$. Cell viability was then assessed using a 3-(4,5-dimethyl-2-thiazolyl)2,5-diphenyl-2H-tetrazolium bromide (MTT) assay according to the manufacturer's instructions (Sigma, St Louis, MO, USA). The absorbance of each well was read by a microplate manager (Bio-Rad Laboratories, Inc. USA) at 550 $\mathrm{nm}$. 


\subsection{Transwell cell migration assay}

A transwell cell migration assay with human RPE cells was performed using a modified Boyden chamber model (Transwell apparatus, $8.0-\mu \mathrm{m}$ pore size, Falcon). For detecting RPE cell migration in the Transwell, the polyethylene terephthalate membrane insert was coated with fibronectin $(25 \mu \mathrm{g} / \mathrm{mL})$ for $1 \mathrm{~h}$. RPE cells were pre-treated with TM $(100 \mathrm{ng} / \mathrm{mL})$ or PBS (Ctrl) for $24 \mathrm{~h}$ and were then trypsinized and resuspended in $0.5 \%$ FBS DMEM to a concentration of $5.0 \times 10^{5}$ cells $/ \mathrm{mL}$. A $100-\mu \mathrm{L}$ RPE cell suspension (final concentration: $5 \times 10^{4}$ cells/well) was plated into the upper chambers, and the lower chambers were filled with $0.6 \mathrm{~mL}$ of $0.5 \%$ FBS DMEM. After a 1-h incubation period $\left(37{ }^{\circ} \mathrm{C}, 95 \%\right.$ air $\left./ 5 \% \mathrm{CO}_{2}\right)$, PDGF$\mathrm{BB}(25 \mathrm{ng} / \mathrm{mL})$ or PBS was added to the lower chambers to induce chemotaxis. After $5 \mathrm{~h}$ of incubation, Transwell inserts were washed with PBS and fixed with pre-chilled methanol $\left(10 \mathrm{~min}\right.$ at $\left.4{ }^{\circ} \mathrm{C}\right)$. Cells were then stained with hematoxylin, and all non-migrant cells were removed from the upper faces of the Transwell membranes with a cotton swab. Migration was quantified by counting the number of stained cells $/ 10 \times$ in images taken with a scanning microscope (Aperio ScanScope, Leica, Wetzlar, Germany). Four randomly chosen fields were counted per membrane. Each experiment was performed in duplicate and repeated at least three times.

\subsection{Cell scratch assay}

Human RPE cells were cultured in DMEM containing $10 \% \mathrm{FBS}$ in a 12-well cell culture plate. After the cells were cultured to confluence, a straight scratch was made with a $20-\mu \mathrm{L}$ pipette tip. Scratched cells were then washed with PBS once, immediately, and treated with TGF- $\beta 2$ (10 $\mathrm{ng} / \mathrm{mL})$, with or without TM $(100 \mathrm{ng} / \mathrm{mL})$, for $15 \mathrm{~h}$. Images were taken with a microscope (BZ-X700, KEYENCE). Each experiment was performed in duplicate and repeated at least three times.

\subsection{TUNEL assay}

Apoptosis was detected by the terminal deoxynucleotidyl transferase (TdT)-mediated dUTP-biotin nick end-labeling (TUNEL) assay according to the manufacturer's instructions (Promega). Images were taken with a fluorescence microscope (BZ-X700, KEYENCE).

\subsection{Statistical analyses}

All experiments were performed at least three times, and the results are presented as mean \pm SEM. The statistical significance of differences between groups was analyzed using the Tukey HSD test or a two-tailed Student's $t$-test. For comparison with the control group, Bonferroni and Holm tests or two-tailed Student's $t$-tests were applied. One-way analysis of variance with Bonferroni correction for multiple comparisons was performed where applicable. Differences were considered significant at $p<0.05$. Sta- tistical analyses were performed using JMP $®$ version 7.0.1 (SAS Institute, Cary, NC, USA).

\section{Results}

\subsection{ER stress inhibits EMT of RPE cells}

To explore the effects of ER stress on EMT of RPE cells, we used two well-known ER stress inducers, tunicamycin (TM) and thapsigargin (TG), to induce ER stress in RPE cells. Then we examined the expression of an epithelial marker, occludin, and mesenchymal markers, alphasmooth muscle actin ( $\alpha$-SMA) and fibronectin (FN), in RPE cells. As shown in Fig. 1, treatment with TM and TG (for $24 \mathrm{~h}$ ) elicited ER stress in RPE cells in a dose-dependent manner, which was indicated by the gradual increase in the expression of ER stress marker GRP78 $(p<0.05)$. Exposure of RPE cells to different concentrations of TM and TG (for $24 \mathrm{~h}$ ) reduced the expression of $\alpha$-SMA and FN, and increased the expression of occludin significantly $(p<0.05)$. These phenomena were strongly associated with the expression of GRP78 (Fig. 1). These results suggested that ER stress inhibited EMT in RPE cells.

\subsection{Induction of ER stress inhibits TGF- $\beta 2$-induced EMT in RPE cells}

TGF- $\beta 2$, the major TGF- $\beta$ isoform in the posterior eye, is a critical inducer of EMT in RPE cells [14,15]. To investigate whether ER stress can inhibit TGF- $\beta 2$-induced EMT of RPE cells, we examined the expression of epithelial markers and mesenchymal markers in RPE cells at both the mRNA and protein levels. As shown in Fig. 2A and Supplemental Fig. 1, treatment with TGF- $\beta 2(10 \mathrm{ng} / \mathrm{mL})$ significantly upregulated the expression of $\alpha$-SMA and FN, and decreased the expression of occludin at both protein and mRNA levels. This effect was significantly inhibited by a 4-h pre-treatment with $200 \mathrm{ng} / \mathrm{mL}$ and $300 \mathrm{ng} / \mathrm{mL} \mathrm{TM}$. In accordance with the results of western blotting, immunofluorescence showed that $\alpha$-SMA (Fig. 2B) and FN (Fig. 2C) staining were markedly attenuated by $24-\mathrm{h}$ TM treatment; however, immunostaining of ZO-1 with TM treatment (100 $\mathrm{ng} / \mathrm{mL}$ ) didn't appear to be obviously stronger than that of the control (Fig. 2D). Moreover, the staining of $\alpha$-SMA (Fig. 2B) and FN (Fig. 2C) was strongly enhanced by treatment with TGF- $\beta 2$, which was considerably inhibited by TM treatment. Interestingly, not only was ZO-1 expression reduced, but the distribution of ZO-1 staining was changed by TGF- $\beta 2$ treatment (Fig. 2D). Moreover, the morphology of RPE cells changed to a spindle-like shape after TGF$\beta 2$ treatment, according to the distribution of FN, $\alpha$-SMA, and ZO-1, whereas with TM treatment, RPE cells maintained a hexagonal shape, as shown by the staining of $\mathrm{ZO}$ 1 (Fig. 2D). Taken together, these results implied that induction of ER stress could inhibit TGF- $\beta 2$-induced EMT of RPE cells. 
A
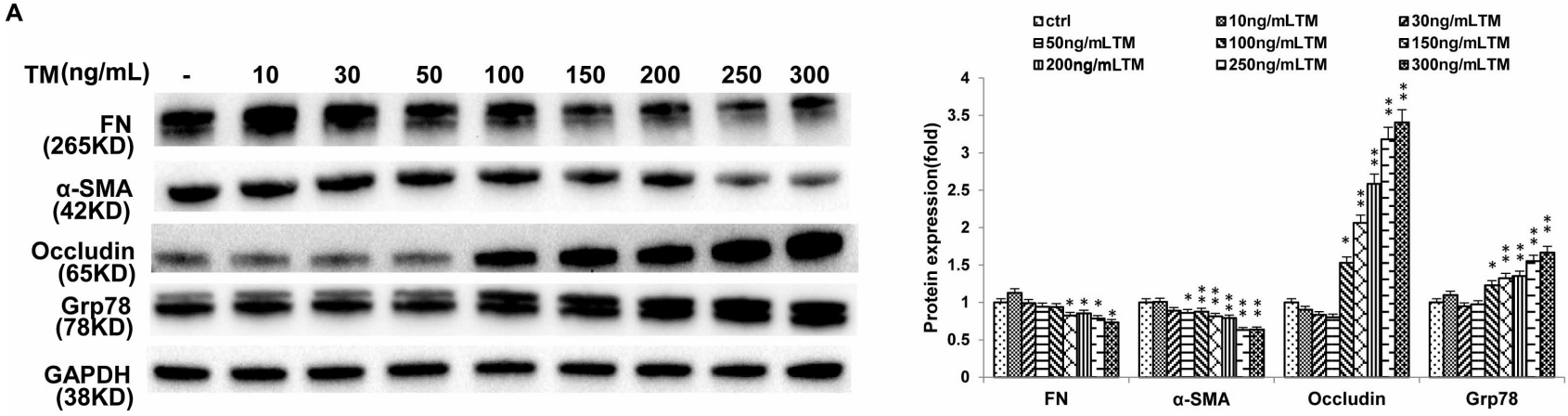

B
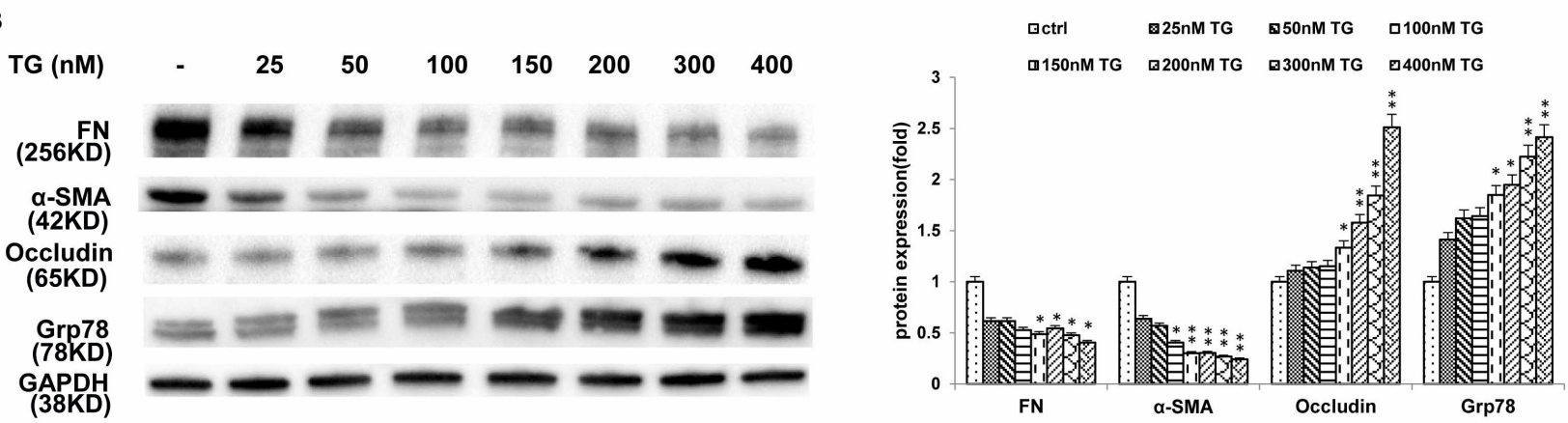

Fig. 1. ER stress inhibits epithelial to mesenchymal transition in a dose-dependent manner in human RPE cells. The human RPE cells were treated with different concentrations of TM $(0-300 \mathrm{ng} / \mathrm{mL})(\mathrm{A})$ or TG $(0-400 \mathrm{nM})(\mathrm{B})$ for $24 \mathrm{~h}$, and the expression of $\alpha$-smooth muscle actin (SMA), fibronectin (FN), occludin, and Grp78 was analyzed by western blot. GAPDH was used as the internal control. Data are presented as mean \pm SEM. $\mathrm{n}=4$ /group. ${ }^{*} p<0.05,{ }^{* *} p<0.01$ vs. Ctrl. Densitometry results of western blotting are shown on the right of each blot; GAPDH was used as a protein-loading control.

\subsection{Effects of ER stress on TGF- $\beta$ signaling}

The TGF- $\beta$ signaling pathway is one of the bestrecognized pathways that promotes EMT in various cell types, including RPE cells $[16,17]$. Therefore, we further examined the effects of ER stress on the expression and phosphorylation of the TGF- $\beta$ receptor 2, SMAD2, and SMAD3, induced by TGF- $\beta$ in RPE cells. As shown in Fig. 3, TGF- $\beta_{2}(10 \mathrm{ng} / \mathrm{mL}, 30 \mathrm{~min})$ significantly induced the phosphorylation of TGF- $\beta$ receptor 2 and SMAD2/3 ( $p$ $<0.01$ ), which were markedly inhibited by pre-treatment with TM for $16 \mathrm{~h}(p<0.01)$. Interestingly, TM treatment alone could also inhibit the expression of TGF- $\beta$ receptor 2 and SMAD2/3 at the protein level $(p<0.05)$. The results of quantitative polymerase chain reaction (RT-qPCR) further validated the effects of ER stress on the expression of TGF $-\beta$ receptor 2 and SMAD3; however, the expression of SMAD2 was not inhibited by TM treatment at the mRNA level (Supplementary Fig. 2). These results suggest that ER stress could inhibit the EMT of RPE cells by inactivating TGF- $\beta$ signaling.

\subsection{ER stress inhibits RPE cells migration}

During the process of EMT and formation of subretinal fibrosis, one of the most important functional characteristic changes of the RPE cells is the increase of migration.
Therefore, transwell migration assay and scratch assay were used to investigate the changes in the migratory capacity of RPE cells. PDGF-BB, an important growth factor that is expressed in the subretinal membrane in PVR and lesions of nAMD and is associated with the migration of RPE cells, was used to induce the cell migration [18]. As shown in Fig. 4A, PDGF-BB significantly stimulated the migration of RPE cells, whereas TM treatment significantly inhibited the migration of RPE cells $(>60 \%)$, with or without the stimulation of PDGF-BB $(p<0.01)$. The results of the scratch assay further validated the inhibitory effect of ER stress on RPE cell migration, as shown in Fig. 4B. These data suggest that TM-induced ER stress could inhibit the migration of RPE cells.

\subsection{Effects of high concentrations of TM and TG on RPE cell survival and apoptosis}

To explore the effects of TM and TG on cell viability and apoptosis in RPE cells at the concentration we used in this study, we conducted an MTT assay and a TUNEL assay. In the MTT assay, lower concentrations of TM (up to $300 \mathrm{ng} / \mathrm{mL}$ ) and TG (up to $250 \mathrm{nM}$ ) revealed no cell toxicity in RPE cells; however, high concentrations of TM $(\geq 400 \mathrm{ng} / \mathrm{mL})$ or TG $(\geq 300 \mathrm{nM})$ reduced the viability of RPE cells. In addition, TM treatment induced apoptosis of RPE cells in a dose-dependent manner, as shown in the 


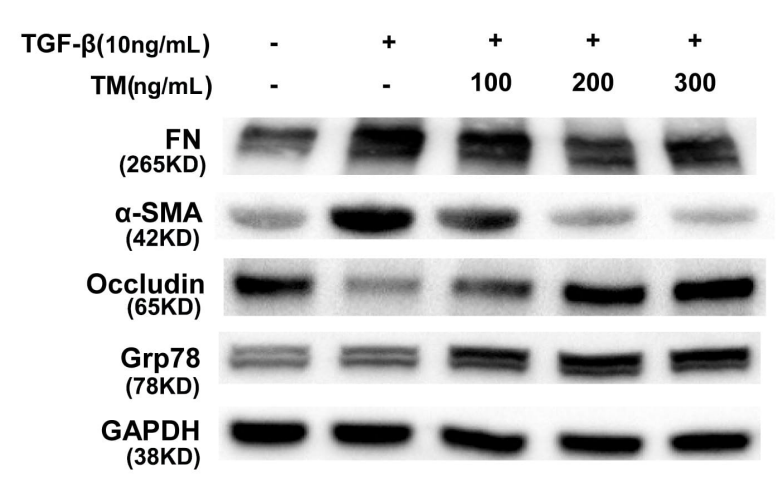

口Ctrl

$\checkmark 200 \mathrm{ng} / \mathrm{mLTM}+\mathrm{TGF}-\beta 2 \square 300 \mathrm{ng} / \mathrm{mLTM}+\mathrm{TGF}-\beta 2$

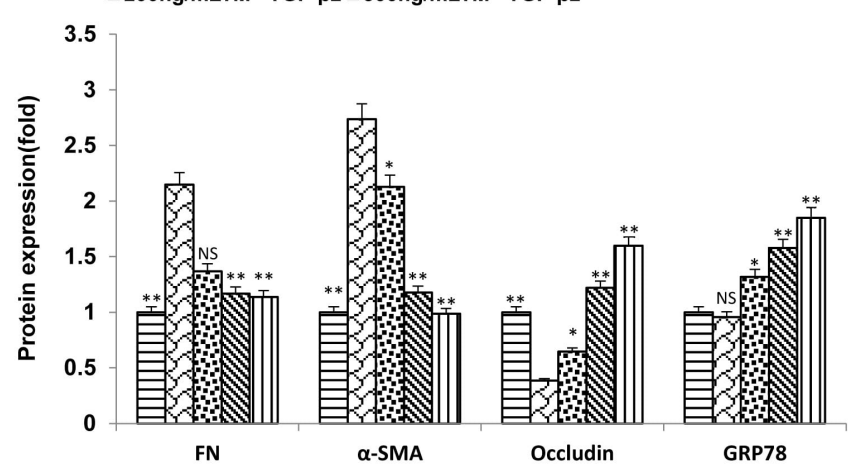

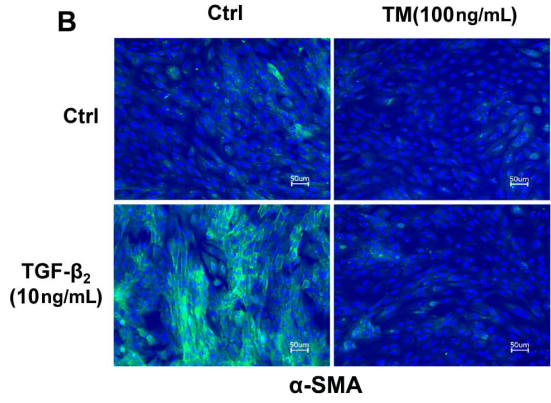
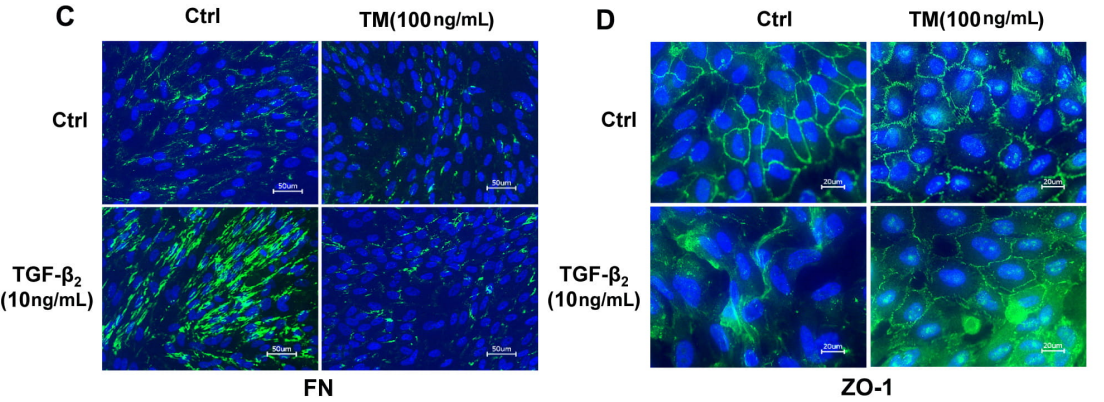

Fig. 2. ER stress inhibits TGF- $\beta$ 2-induced epithelial to mesenchymal transition in RPE cells. (A) Human RPE cells were pre-treated with or without different concentrations of TM for $4 \mathrm{~h}$ and then treated with TGF- $\beta 2(10 \mathrm{ng} / \mathrm{mL})$ for another $48 \mathrm{~h}$. The expression of $\alpha$-smooth muscle actin (SMA), fibronectin (FN), occludin, and Grp78 proteins was analyzed by western blot. GAPDH was used as internal control. Data are presented as mean \pm SEM. $\mathrm{n}=6$ /group. NS, not significant. ${ }^{*} p<0.05,{ }^{* *} p<0.01$ vs. Ctrl. (B) Human RPE cells were treated with 0 or $100 \mathrm{ng} / \mathrm{mL}$ TM with or without TGF- $\beta 2(10 \mathrm{ng} / \mathrm{mL})$ treatment for $24 \mathrm{~h}$. The immunofluorescence staining for FN is shown in green. Nuclei are stained blue. (C) Human RPE cells were treated with 0 or $100 \mathrm{ng} / \mathrm{mL}$ TM with or without TGF- $\beta 2$ (10 $\mathrm{ng} / \mathrm{mL}$ ) treatment for $24 \mathrm{~h}$. The immunofluorescence staining for $\alpha$-SMA is shown in green. Nuclei are stained blue. (D) Human RPE cells were treated with 0 or $100 \mathrm{ng} / \mathrm{mL}$ TM with or without TGF- $\beta 2(10 \mathrm{ng} / \mathrm{mL})$ treatment for $24 \mathrm{~h}$. The immunofluorescence staining for zonula occludens-1 (ZO-1) is shown in green. Nuclei are stained blue. Scale bar: $50 \mu \mathrm{m}$ for FN and $\alpha$-SMA, $20 \mu \mathrm{m}$ for ZO-1.
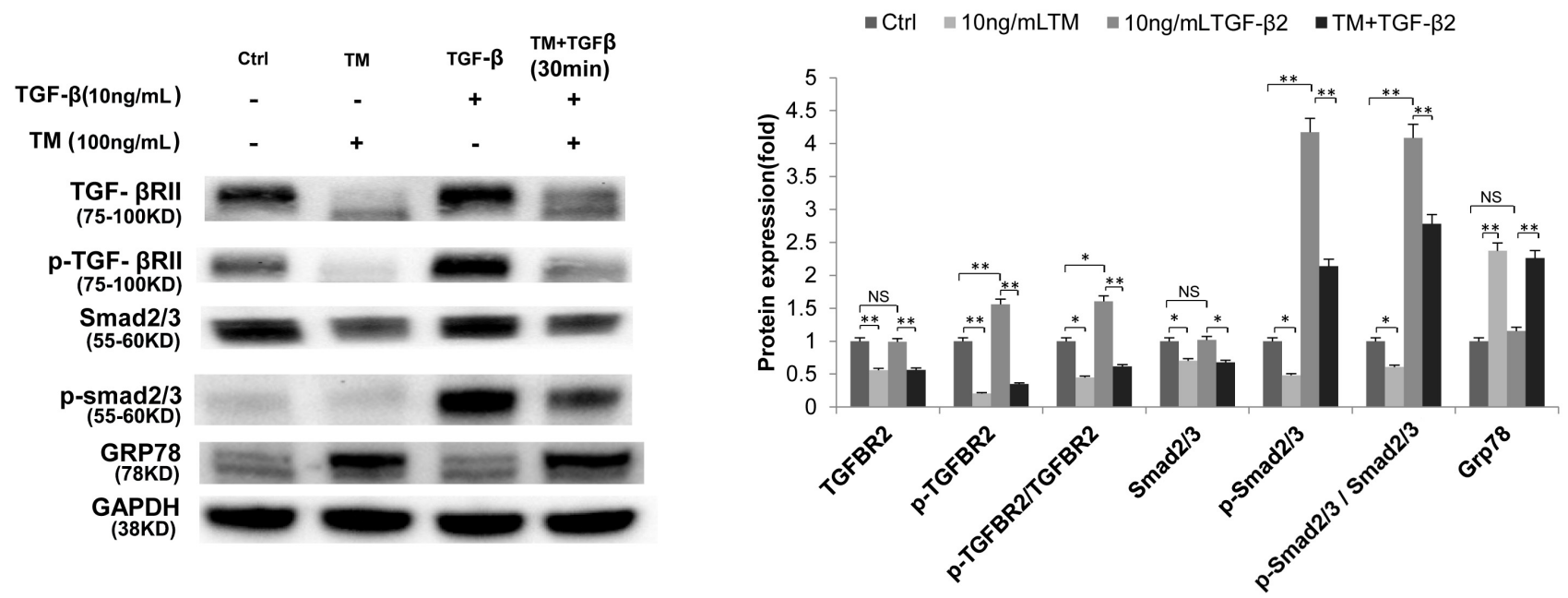

Fig. 3. Effect of ER stress on TGF- $\beta$ signaling in human RPE cells. The human RPE cells were pre-treated with 0 or $100 \mathrm{ng} / \mathrm{mL} \mathrm{TM}$ for $24 \mathrm{~h}$ and then treated with or without $10 \mathrm{ng} / \mathrm{mL}$ TGF- $\beta 2$ for another $30 \mathrm{~min}$. The expressions of TGF- $\beta$ R2, p-TGF- $\beta$ R2, SMAD2/3, p-SMAD2/3, and GRP78 proteins were analyzed by western blot. GAPDH was used as internal control. NS, not significant. ${ }^{*} p<0.05$, ${ }^{* *} p<0.01$. Data are presented as mean \pm SEM. 
A

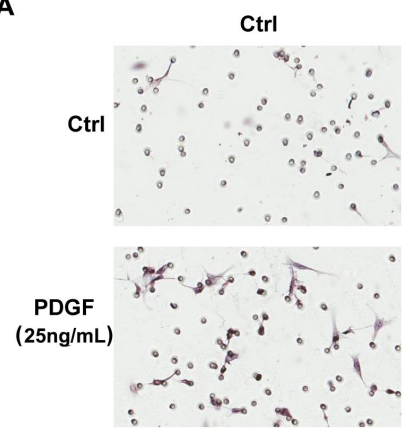

B

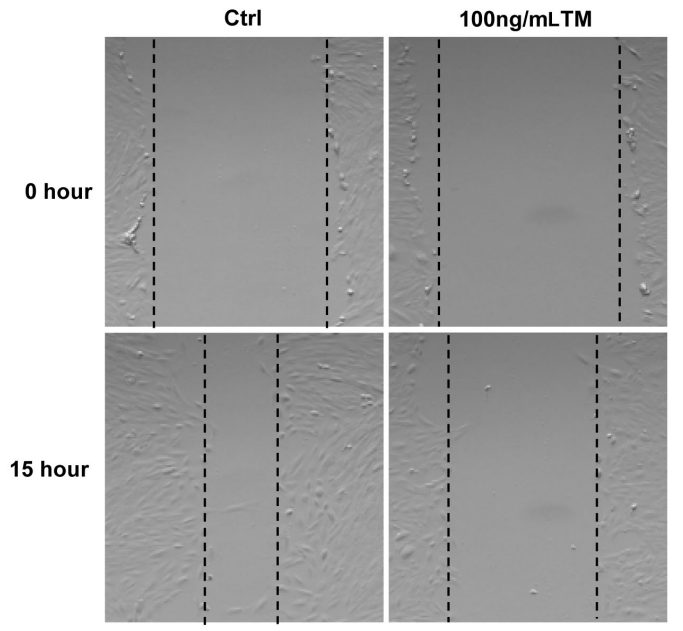

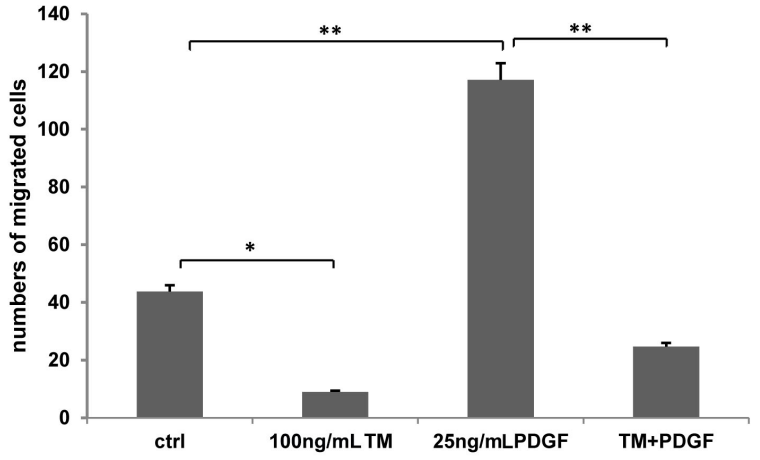

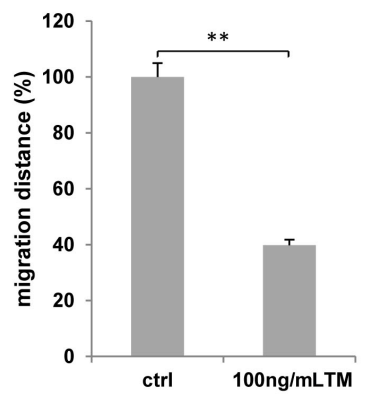

Fig. 4. ER stress inhibits migration of human RPE cells. (A) The human RPE cells were treated with or without $100 \mathrm{ng} / \mathrm{mL} \mathrm{TM}$ for $24 \mathrm{~h}$, after which the cells were treated with or without $25 \mathrm{ng} / \mathrm{mL}$ PDGF for $4 \mathrm{~h}$. RPE cell migration was detected by Boyden chamber migration assay. (B) Human RPE cells were pre-treated with 0 or $100 \mathrm{ng} / \mathrm{mL}$ TM for $8 \mathrm{~h}$ and were then scratched for the scratch assay. NS, not significant. ${ }^{*} p<0.05,{ }^{* *} p<0.01$. Data are presented as mean $\pm \mathrm{SEM}$.

TUNEL assay (Supplementary Fig. 3). These data indicated that mild ER stress does not affect cell survival in RPE cells; however, severe ER stress inhibits cell survival and induces apoptosis of RPE cells.

\section{Discussion}

To the best of our knowledge, this study demonstrates for the first time that chemical induction of ER stress by TM or TG inhibited TGF- $\beta 2$-induced EMT of RPE cells. We further confirmed that ER stress inactivated TGF- $\beta$ signaling and inhibited migration of RPE cells. We also revealed that mild ER stress does not affect the viability of RPE cells; however, severe ER stress induced by higher concentrations of TM and TG could inhibit cell survival and induce apoptosis of RPE cells. These results provide evidence for the association between ER stress and EMT in RPE cells and suggest a potential role of ER stress as a modifiable target for the prevention or treatment of fibrotic retinal diseases, such as PVR and AMD, considering that ER stress is an important mediator in the pathogenesis of such diseases.

According to its different functional consequences, EMT can be divided into three different subtypes. Type I EMT is associated with implantation, embryogenesis, and organ formation; Type II EMT is known to play a critical role in wound healing, tissue regeneration or reconstruction, and organ fibrosis; while Type III EMT usually occurs in neoplastic cells during the cancer progress and metastasis [19]. In the development of PVR or in late-stage AMD, RPE cells undergo EMT and transdifferentiate into a fibroblastic phenotype characterized by increased EMT phenotypes and the ability to proliferate and migrate to facilitate development of subretinal fibrosis. This process is regulated by the participation of a number of growth factors and cytokines, such as TNF, HGF, PDGF, and TGF- $\beta$. TGF- $\beta$ is a multifunctional growth factor and a major inducer of EMT in the pathogenesis of fibrotic eye diseases $[20,21]$. Overexpressed TGF- $\beta$ is found in the vitreous of patients with PVR, which is highly related to the severity of the disease $[22,23]$. Since TGF- $\beta 2$ is the predominant isoform of TGF- $\beta$ in the posterior eye, we used TGF- $\beta 2$ to induce EMT in RPE cells in our study. Consistent with previous studies, our results confirmed that RPE cells undergo EMT when stimulated with TGF- $\beta 2$, as shown by a morphological change with up-regulation of $\alpha$-SMA and FN, and downregulation of occludin at both protein and mRNA levels. There are several signaling pathways that are in- 
volved in regulating the process of EMT. Among them, the best known is the TGF- $\beta$ signaling pathway, which signals through canonical SMAD signaling as well as through some non-SMAD-dependent pathways, such as the MAPK pathway and PI-3 kinase pathway. In the canonical SMAD signaling pathway, signaling is activated by the interaction of TGF- $\beta$ with type II and type I serine-threonine kinase receptors, termed TGF- $\beta$ RII and TGF- $\beta$ RI. Following ligand binding, TGF- $\beta$ RI is phosphorylated by TGF- $\beta$ RII, which in turn phosphorylates SMAD2/3. Activated SMAD2/3 then binds to SMAD4 and translocated into the nucleus to regulate the expression of target genes. Numerous studies have revealed that the SMAD signaling pathway is critically involved in EMT and in the pathogenesis of tissue fibrosis [24,25]. Inactivation of SMAD signaling significantly inhibits EMT in RPE cells and fibrogenesis in many other cell types [24,26,27]. Extensive studies have revealed the essential role of SMAD3 in EMT and fibrosis [28]. RPE cells in Smad3-null mice failed to undergo EMT in response to retinal detachment, indicating the pivotal role of SMAD3 in the EMT of RPE cells [29]. Furthermore, Shin et al. [11] demonstrated that ER stress preconditioning blocked activation of SMAD signaling in human peritoneal mesothelial cells. In this study, we found that ER stress inducers inhibited the phosphorylation of SMAD2/3 induced by TGF- $\beta 2$ treatment of RPE cells. In addition, it also inhibited the expression of TGF- $\beta$ receptor and SMAD3 with or without TGF- $\beta$ stimulation. These results support the suggestion that ER stress inhibits EMT of RPE cells, at least in part, by inactivating the TGF $\beta /$ SMAD signaling pathway.

The ER is an important organelle that is essential for the synthesis of lipids, regulation of calcium homeostasis, and most importantly, the synthesis, folding, assembly, modification, and translocation of proteins. Under normal conditions, unfolded or misfolded proteins are degraded via ER-associated degradation, while under harsh environmental stresses, such as hypoxia, nutrient deprivation, or metabolic stress, accumulation of misfolded/unfolded proteins in the ER lumen leads to activation of ER stress and the UPR. GRP78 is one of the most important and predominant ER chaperones that identify misfolded proteins and facilitate protein folding in the ER. Numerous studies have verified the specific induction of GRP78 as an indicator of ER stress [30]. In our study, both TM and TG, two classical chemical inducers of ER stress, increased the expression of GRP78 in a dose-dependent manner. Over the past decade, evidence has emerged to reveal an association between ER stress and a variety of human diseases, including metabolic disease, neurodegenerative disorders, infectious and inflammatory diseases, fibrosis, and cancer [8]; however, the exact relationship between ER stress and those diseases has not been fully understood. On the one hand, a number of studies have demonstrated that ER stress can suppress EMT and fibrosis. For example, downregulation of GRP78 promotes EMT and migration of human hepatocellular carcinoma cells [31]. Knockdown of GRP78 induces EMT in colon cancer cells and Mahlavu cells, as characterized by downregulation of vimentin, upregulation of E-cadherin, and increased migratory ability [32,33]. A more recent study found that matrine treatment inhibited EMT in human prostate cancer cells as well as activating the ER stress signal pathway in both vivo and vitro [34]. Similar results were observed in Honokiol treated melanoma cells [35], indicating a potential suppressive effect of ER stress on EMT. Furthermore, pre-treatment with TM or TG inhibited TGF- $\beta 1$-induced EMT in HPMCs, suggesting a protective role of ER stress in TGF- $\beta$-induced EMT [11]. On the other hand, ER stress can also play a facilitatory role in EMT and fibrosis. Induction of ER stress results in EMT in human lens epithelial cells and lung epithelial cells [12,36]. Similarly, Moon et al. [37] showed that TM or TG treatment induces EMT in HK-2 cells via activation of the Src pathway. Taken together, these results suggest that the role of ER stress in EMT and fibrosis may be contextdependent meaning based on difference of microenvironment, even though the exact causes of the discrepancies among these studies remain unclear. We speculate that the differences in cell types, cell status, culture conditions, and the concentration of TM or TG used in each experiment might be possible reasons.

Despite ample studies showing the association of ER stress and EMT with organ fibrosis, evidence regarding the effects of ER stress on EMT in RPE cells remains very limited. Yoshikawa et al. [38] reported that ER stress increased the expression of tight junctions, such as occludin, claudin-1, and ZO-1, at both the protein and mRNA levels in APRE-19 cells. Another study showed that overexpression of the ER protein 29 (ERp29), which is increased during ER stress, protected against the CSE-induced reduction in ZO-1 expression in ARPE-19 cells [39]. However, no previous studies have explored the role of ER stress on EMT induced by TGF- $\beta$ in human RPE cells. Here, we reported that chemical induction of ER stress in RPE cells, by means of TM or TG, increased the expression of the tight junction protein, occludin, and decreased the expression of the mesenchymal markers, $\alpha$-SMA and FN. Moreover, ER stress could also inhibit TGF- $\beta$-induced EMT and inactivate SMAD signaling. These results suggest the beneficial effects of ER stress on the EMT of RPE cells.

Proliferation and migration of RPE cells are key abnormal steps in the formation of subretinal or epiretinal fibrosis membranes during the development of PVR or nAMD [5,40]. Previous studies have shown that, when RPE cells undergo EMT, the dedifferentiated cells acquire an increased migratory capacity and migrate to the vitreous cavity to contribute to the formation of the contractile membranes $[1,41,42]$. This process is facilitated by the remodeling of the ECM, involving proteins such as fibronectin and collagen $[1,43]$. While several studies have shown the inhibitory effect of ER stress on cell migration, no study 
investigated its effect on cell migration in RPE cells. In vascular smooth muscle cells, TM pretreatment inhibited PDFG-BB-induced cell migration [44]. Downregulation of GRP78 increased the migratory ability of colon cancer cells and Mahlava cells $[32,33]$. In our study, consistent with its inhibitory effect on EMT, ER stress induced by TM significantly reduced cell migration and fibronectin synthesis in RPE cells, without causing significant apoptosis. Moreover, it could also inhibit PDGF-BB-induced cell migration. These results indicate that ER stress might regulate ECM remodeling and RPE cell migration, not only through EMT inhibition but also by mediating signaling pathways involved in PDGF-BB-induced migration.

\section{Conclusions}

In conclusion, our data demonstrated that ER stress inhibits TGF- $\beta$-induced EMT in RPE cells. Our results suggest the potential role of ER stress in the pathogenesis of intraocular fibrotic diseases, such as PVR and AMD and may be as a new therapeutic approach to prevent such kind of disorder.

\section{Abbreviations}

ER stress, Endoplasmic reticulum stress; EMT, Epithelial-mesenchymal transition; UPR, Unfolded protein response; RPE, Retinal pigment epithelial; PVR, Proliferative vitreoretinopathy; AMD, Age-related macular degeneration; TGF- $\beta$, Transforming growth factor- $\beta$; TM, Tunicamycin; TG, Thapsigargin; FN, Fibronectin; $\alpha$-SMA, Alpha-smooth muscle actin; ZO-1, Zonula occludens-1; GRP78, Glucose regulated p rotein $78 \mathrm{kD}$; PERK, Protein kinase R-like ER kinase; ATF6, Activating transcriptional factor 6; ATF4, Activating transcriptional factor 4; TGFBR2, Transforming growth factor- $\beta$ receptor II; GAPDH, Glyceralehyde-3-phosphate dehydrogenase.

\section{Author contributions}

$\mathrm{SO}, \mathrm{SH}$ and $\mathrm{XX}$ conceived and designed the experiments; SO performed the experiments; SO, DJ analyzed data; $\mathrm{SO}, \mathrm{SH}$ and $\mathrm{XX}$ involved in writing and reviewing the paper, and all authors had final approval of the submitted versions.

\section{Ethics approval and consent to participate}

Not applicable.

\section{Acknowledgment}

The research is supported by a fellowship from the second Xiangya Hospital of Xiangya School of Medicine of Central South University for Research Abroad. The authors thank Christine K. Spee, Eric Barron and Ernesto Barron for technical assistance.

\section{Funding}

This research received no external funding.

\section{Conflict of interest}

The authors declare no conflict of interest. SH is serving as one of the Guest Editor of this journal. We declare that $\mathrm{SH}$ had no involvement in the peer review of this article and has no access to information regarding its peer review. Full responsibility for the editorial process for this article was delegated to GP.

\section{Supplementary material}

Supplementary material associated with this article can be found, in the online version, at https://www.imrpre ss.com/journal/FBL/27/2/10.31083/j.fbl2702038.

\section{References}

[1] Ishikawa K, Kannan R, Hinton DR. Molecular mechanisms of subretinal fibrosis in age-related macular degeneration. Experimental Eye Research. 2016; 142: 19-25.

[2] Hiscott P, Sheridan C, Magee RM, Grierson I. Matrix and the retinal pigment epithelium in proliferative retinal disease. Progress in Retinal and Eye Research. 1999; 18: 167-190.

[3] Friedlander M. Fibrosis and diseases of the eye. The Journal of Clinical Investigation. 2007; 117: 576-586.

[4] Stone RC, Pastar I, Ojeh N, Chen V, Liu S, Garzon KI, et al. Epithelial-mesenchymal transition in tissue repair and fibrosis. Cell and Tissue Research. 2016; 365: 495-506.

[5] Ishikawa K, Sreekumar PG, Spee C, Nazari H, Zhu D, Kannan $\mathrm{R}$, et al. AB-Crystallin Regulates Subretinal Fibrosis by Modulation of Epithelial-Mesenchymal Transition. the American Journal of Pathology. 2016; 186: 859-873.

[6] Mudhar HS. A brief review of the histopathology of proliferative vitreoretinopathy (PVR). Eye. 2020; 34: 246-250.

[7] Tenbrock L, Wolf J, Boneva S, Schlecht A, Agostini H, Wieghofer $\mathrm{P}$, et al. Subretinal fibrosis in neovascular age-related macular degeneration: current concepts, therapeutic avenues, and future perspectives. Cell and Tissue Research. 2021. (in press)

[8] Kropski JA, Blackwell TS. Endoplasmic reticulum stress in the pathogenesis of fibrotic disease. Journal of Clinical Investigation. 2018; 128: 64-73.

[9] Hsu H, Liu C, Lin J, Hsu T, Hsu J, Su K, et al. Involvement of ER stress, PI3K/AKT activation, and lung fibroblast proliferation in bleomycin-induced pulmonary fibrosis. Scientific Reports. 2017; 7: 14272.

[10] Fu L, Zhao H, Xiang Y, Xiang H, Hu B, Tan Z, et al. Reactive oxygen species-evoked endoplasmic reticulum stress mediates 1-nitropyrene-induced epithelial-mesenchymal transition and pulmonary fibrosis. Environmental Pollution. 2021; 283: 117134.

[11] Shin H, Ryu E, Oh E, Kang D. Endoplasmic reticulum stress as a novel target to ameliorate epithelial-to-mesenchymal transition and apoptosis of human peritoneal mesothelial cells. Laboratory Investigation. 2015; 95: 1157-1173.

[12] Zhou S, Yang J, Wang M, Zheng D, Liu Y. Endoplasmic reticulum stress regulates epithelialmesenchymal transition in human lens epithelial cells. Molecular Medicine Reports. 2020; 21: 173-180.

[13] Sonoda S, Spee C, Barron E, Ryan SJ, Kannan R, Hinton DR. A protocol for the culture and differentiation of highly polarized 
human retinal pigment epithelial cells. Nature Protocols. 2009; 4: 662-673.

[14] Pfeffer BA, Flanders KC, Guérin CJ, Danielpour D, Anderson $\mathrm{DH}$. Transforming growth factor beta 2 is the predominant isoform in the neural retina, retinal pigment epithelium-choroid and vitreous of the monkey eye. Experimental Eye Research. 1994; 59: $323-333$

[15] Feng Z, Li R, Shi H, Bi W, Hou W, Zhang X. Combined silencing of TGF-beta2 and Snail genes inhibit epithelialmesenchymal transition of retinal pigment epithelial cells under hypoxia. Graefe's Archive for Clinical and Experimental Ophthalmology. 2015; 253: 875-884.

[16] Dongre A, Weinberg RA. New insights into the mechanisms of epithelial-mesenchymal transition and implications for cancer. Nature Reviews Molecular Cell Biology. 2019; 20: 69-84.

[17] Zhou M, Geathers JS, Grillo SL, Weber SR, Wang W, Zhao Y, et al. Sundstrom: Role of Epithelial-Mesenchymal Transition in Retinal Pigment Epithelium Dysfunction. Frontiers in Cell and Developmental Biology. 2020; 8: 501.

[18] Zhang H, Shang Q, An J, Wang C, Ma J. Crocetin inhibits PDGFBB-induced proliferation and migration of retinal pigment epithelial cells. European Journal of Pharmacology. 2019; 842: 329-337.

[19] Kalluri R, Weinberg RA. The basics of epithelial-mesenchymal transition. The Journal of Clinical Investigation. 2009; 119: 1420-1428.

[20] Saika S, Yamanaka O, Okada Y, Tanaka S, Miyamoto T, Sumioka T, et al. Shirai and K. Ikeda: TGF beta in fibroproliferative diseases in the eye. Frontiers in Bioscience (Scholar edition). 2009; 1: 376-390.

[21] Gamulescu M, Chen Y, He S, Spee C, Jin M, Ryan SJ, et al. Transforming growth factor beta2-induced myofibroblastic differentiation of human retinal pigment epithelial cells: regulation by extracellular matrix proteins and hepatocyte growth factor. Experimental Eye Research. 2006; 83: 212-222.

[22] Kita T, Hata Y, Arita R, Kawahara S, Miura M, Nakao S, et al. Role of TGF-beta in proliferative vitreoretinal diseases and ROCK as a therapeutic target. Proceedings of the National Academy of Sciences of the United States of America. 2008; 105: 17504-17509.

[23] Connor TB, Roberts AB, Sporn MB, Danielpour D, Dart LL, Michels RG, et al. Correlation of fibrosis and transforming growth factor-beta type 2 levels in the eye. The Journal of Clinical Investigation. 1989; 83: 1661-1666.

[24] Frangogiannis NG. Transforming growth factor- $\beta$ in tissue fibrosis. Journal of Experimental Medicine. 2020; 217: e20190103.

[25] Xu J, Lamouille S, Derynck R. TGF-beta-induced epithelial to mesenchymal transition. Cell Research. 2009; 19: 156-172.

[26] Chen CL, Chen YH, Tai MC, Liang CM, Lu DW, Chen JT. Resveratrol inhibits transforming growth factor-beta2-induced epithelial-to-mesenchymal transition in human retinal pigment epithelial cells by suppressing the Smad pathway. Drug, Design, Development and Therapy. 2017; 11: 163-173.

[27] Geng X, Ma A, He J, Wang L, Jia Y, Shao G, et al. Ganoderic acid hinders renal fibrosis via suppressing the TGF- $\beta /$ Smad and MAPK signaling pathways. Acta Pharmacologica Sinica. 2020; 41: 670-677.

[28] Roberts AB, Tian F, Byfield SD, Stuelten C, Ooshima A, Saika $\mathrm{S}$, et al. Smad3 is key to TGF-beta-mediated epithelial-tomesenchymal transition, fibrosis, tumor suppression and metastasis. Cytokine \& Growth Factor Reviews. 2006; 17: 19-27.

[29] Saika S, Kono-Saika S, Tanaka T, Yamanaka O, Ohnishi Y, Sato $\mathrm{M}$, et al. Smad3 is required for dedifferentiation of retinal pig- ment epithelium following retinal detachment in mice. Laboratory Investigation. 2004; 84: 1245-1258.

[30] Lee AS. The ER chaperone and signaling regulator GRP78/BiP as a monitor of endoplasmic reticulum stress. Methods. 2005; 35: 373-381.

[31] Xia W, Zhuang J, Wang G, Ni J, Wang J, Ye Y. P4HB promotes HCC tumorigenesis through downregulation of GRP78 and subsequent upregulation of epithelial-to-mesenchymal transition. Oncotarget. 2017; 8: 8512-8521.

[32] Chang Y, Chen W, Huang C, Liu H, Wei P. Glucose-regulated protein 78 (GRP78) regulates colon cancer metastasis through EMT biomarkers and the NRF-2/HO-1 pathway. Tumour Biology. 2015 ; 36: 1859-1869.

[33] Tai C, Chin-Sheng H, Kuo L, Wei P, Lu H, Chen $\mathrm{H}$, et al. Survivin-mediated cancer cell migration through GRP78 and epithelial-mesenchymal transition (EMT) marker expression in Mahlavu cells. Annals of Surgical Oncology. 2012; 19: 336343.

[34] Chang J, Hu S, Wang W, Li Y, Zhi W, Lu S, et al. Matrine inhibits prostate cancer via activation of the unfolded protein response/endoplasmic reticulum stress signaling and reversal of epithelial to mesenchymal transition. Molecular Medicine Reports. 2018; 18: 945-957.

[35] Chiu C, Tsai C, Hsieh M, Tsai S, Jan Y, Lin W, et al. Exploiting Honokiol-induced ER stress CHOP activation inhibits the growth and metastasis of melanoma by suppressing the MITF and $\beta$-catenin pathways. Cancer Letters. 2019; 442: 113-125.

[36] Liu D, Zhu H, Gong L, Pu S, Wu Y, Zhang W, et al. Histone Deacetylases Promote ER Stress Induced Epithelial Mesenchymal Transition in Human Lung Epithelial Cells. Cellular Physiology and Biochemistry. 2018; 46: 1821-1834.

[37] Moon SY, Kim HS, Nho KW, Jang YJ, Lee SK. Endoplasmic reticulum stress induces epithelial-mesenchymal transition through autophagy via activation of c-Src kinase. Nephron. Experimental Nephrology. 2014; 126: 127-140.

[38] Yoshikawa T, Ogata N, Izuta H, Shimazawa M, Hara H, Takahashi K. Increased expression of tight junctions in ARPE-19 cells under endoplasmic reticulum stress. Current Eye Research. 2011; 36: 1153-1163.

[39] Huang C, Wang JJ, Jing G, Li J, Jin C, Yu Q, et al. Erp29 Attenuates Cigarette Smoke Extract-Induced Endoplasmic Reticulum Stress and Mitigates Tight Junction Damage in Retinal Pigment Epithelial Cells. Investigative Ophthalmology \& Visual Science. 2015; 56: 6196-6207.

[40] Tosi GM, Marigliani D, Romeo N, Toti P. Disease pathways in proliferative vitreoretinopathy: an ongoing challenge. Journal of Cellular Physiology. 2014; 229: 1577-1583.

[41] Ishikawa K, He S, Terasaki H, Nazari H, Zhang H, Spee C, et al. Resveratrol inhibits epithelial-mesenchymal transition of retinal pigment epithelium and development of proliferative vitreoretinopathy. Scientific Reports. 2015; 5: 16386.

[42] Tamiya S, Kaplan HJ. Role of epithelial-mesenchymal transition in proliferative vitreoretinopathy. Experimental Eye Research. 2016; 142: 26-31.

[43] Sharma M, Tiwari A, Sharma S, Bhoria P, Gupta V, Gupta A, et al. Fibrotic remodeling of the extracellular matrix through a novel (engineered, dual-function) antibody reactive to a cryptic epitope on the N-terminal $30 \mathrm{kDa}$ fragment of fibronectin. PLoS ONE. 2013; 8: e69343.

[44] Yi N, Chen S, Ma A, Chen P, Yao B, Liang T, et al. Tunicamycin Inhibits PDGF-BB-Induced Proliferation and Migration of Vascular Smooth Muscle Cells through Induction of HO1. the Anatomical Record: Advances in Integrative Anatomy and Evolutionary Biology. 2012; 295: 1462-1472. 